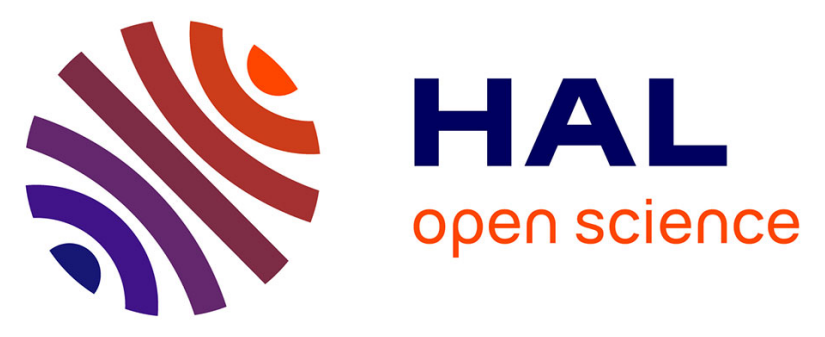

\title{
Virtual Promenade: A New Serious Game for the Rehabilitation of Older Adults with Post-fall Syndrome
}

Pierre Wargnier, Edmond Phuong, Kévin Marivan, Samuel Benveniste, Frédéric Bloch, Serge Reingewirtz, Gilles Kemoun, Anne-Sophie Rigaud

\section{- To cite this version:}

Pierre Wargnier, Edmond Phuong, Kévin Marivan, Samuel Benveniste, Frédéric Bloch, et al.. Virtual Promenade: A New Serious Game for the Rehabilitation of Older Adults with Post-fall Syndrome. 4th International Conference on Serious Games and Applications for Health (IEEE SeGAH 2016), May 2016, Orlando, United States. pp.1-8. hal-01266471

\section{HAL Id: hal-01266471}

https://hal-mines-paristech.archives-ouvertes.fr/hal-01266471

Submitted on 2 Feb 2016

HAL is a multi-disciplinary open access archive for the deposit and dissemination of scientific research documents, whether they are published or not. The documents may come from teaching and research institutions in France or abroad, or from public or private research centers.
L'archive ouverte pluridisciplinaire HAL, est destinée au dépôt et à la diffusion de documents scientifiques de niveau recherche, publiés ou non, émanant des établissements d'enseignement et de recherche français ou étrangers, des laboratoires publics ou privés. 


\title{
Virtual Promenade: A New Serious Game for the Rehabilitation of Older Adults with Post-fall Syndrome
}

\author{
Pierre Wargnier ${ }^{*} \dagger$, Edmond Phuong ${ }^{\ddagger}$, Kévin Marivan ${ }^{\ddagger}$, Samuel Benveniste ${ }^{*} \ddagger$, \\ Frédéric Bloch ${ }^{\dagger} \S$, Serge Reingewirtz ${ }^{\dagger} \ddagger$, Gilles Kemoun ${ }^{\uparrow}$ and Anne-Sophie Rigaud ${ }^{\dagger} \S$ \\ * MINES ParisTech, PSL Research University, Paris, FRANCE, (name.surname@mines-paristech.fr) \\ $\dagger$ Université Paris Descartes Paris, FRANCE, (name.surname@parisdescartes.fr) \\ $\ddagger$ Centre d’Expertise National en Stimulation Cognitive Paris, FRANCE, (name.surname@censtimco.org) \\ $\S$ Broca Hospital, Assistance Publique - Hôpitaux de Paris, Paris, FRANCE (name.surname@aphp. fr) \\ ฯ Université de Poitiers, Poitiers, FRANCE
}

\begin{abstract}
We introduce a novel rehabilitation tool to treat Post-Fall Syndrome (PFS) in older adults: a serious game, called Virtual Promenade, combined with a haptic chair imitating the hips movements of human walk. We report on the user-centered design of our prototype, following "living lab" principles, which was well received by our test participants. This system aims at addressing the psycho-motor consequences of older adults' falls; they are often neglected in current post-fall care practices. We first checked for feasibility and tolerability of such interventions. We then applied a living lab participatory design approach, involving health care professionals and older adults, to build the Virtual Promenade prototype. We found that patients with PFS tolerated the system well and that there were no major obstacles to feasibility. We also report that the aesthetics of the virtual environment is an important motivational factor for older adults and discuss our results in searching for the most suitable game controller for this type of patients and game. Finally, we observed that the chairs' movements improved the immersion in the game.
\end{abstract}

Index Terms-Ergonomics, falls, haptics, living lab, older adults, patient rehabilitation, virtual reality

\section{INTRODUCTION}

Falls, in older adults, are now widely recognized as a serious public health issue, as they may result in dramatic complications that can affect patients' quality of life and autonomy. So far, works on rehabilitation after falls have mostly focused on patients who have post-fall fractures because they can cause various degrees of disability and expose the patient to a highier risk of falling again [1]. However, not paying attention to the psychological consequences of falls may be prejudicial, as these symptoms make motor rehabilitation more difficult. In addition, people who suffered falls may be afraid of going out and tend to isolate themselves, impacting their mood and cognitive abilities. In 1982, Murphy and Isaacs [2], first introduced the term "Post-Fall Syndrome" (PFS) to identify the specific association of the motor and psychological symptoms related to falls in older adults. PFS is a direct consequence of a fall and is defined by the presence of three symptoms, including anxiety, a worry of repeated falls and what is called "Psychomotor Regression Syndrome" [3] or, more recently, "Psychomotor Disadaptation Syndrome" [4], which consist in acute motor disorders.

The symptoms of PFS can resolve spontaneously or develop into a fear of falling (FOF) [5]-[7] in the following months, causing behavioral disorders and limiting autonomy. FOF, according to this definition, has been recently analyzed in the light of the definition of post-traumatic stress disorder (PTSD), according to the fifth edition of the Diagnostic and Statistical Manual of Mental Disorders (DSM V) [8], as substantial similarities can be found. Indeed, for PTSD to occur, the subject must have been the victim of a traumatic event and his or her response must include intense fear, helplessness or feeling of horror. There is, in this definition, a subjective assessment of the intensity of the trauma, and the focus is on the patient's response, which allows for the possibility of defining a fall as a traumatic event [9]-[11].

By distinguishing this specific method, we are in a position to propose rehabilitation adapted to the specific situation of each patient after a fall. If these psychological symptoms can be compared to PTSD, a worthwhile approach would be to explore the potential of Virtual Reality Exposure (VRE) treatment, since it has been shown to be a useful therapeutic tool to improve motor rehabilitation in combat-related PTSD [12]. VRE has also been shown to be a useful therapeutic tool for the motor rehabilitation of patients after a stroke [13] or presenting walking disorders in chronic diseases, such as Parkinson's disease or multiple sclerosis [14], [15]. Several experiments have also been conducted with VRE to treat various psychological disorders such as acrophobia, arachnophobia, aviophobia, claustrophobia or social phobias [16].

We propose a novel rehabilitation tool for older adults with PFS: a virtual strolling system composed of a serious game and a haptic chair that mimics the hips movements of human walk, called Virtual Promenade. Our system is meant to provide physiotherapists and psychomotricians with a safe seated activity, which would be used prior to standing motor rehabilitation, to treat patients who suffered a fall. We believe this system will help reduce FOF in patients, while stimulating 
their psycho-motor cognitive scheme, thanks to the movements of the haptic chair and mirror neurons activation by the sight of the game avatar walking. In addition, this activity could be performed by patients who have fractures and can not yet participate in standard motor rehabilitation activities, which may shorten their treatment and increase their chances of recovery.

To adapt the system's design to the special needs of older adults, who may be frail or have cognitive impairment, we adopt a living lab design approach. It is based on involving all stakeholders (patients, physicians, physiotherapists) in the design process, to produce a truly adapted design that considers both human factors and care environment constraints [17]-[19].

The remainder of this paper is organized as follows: firstly, we review some of the works on serious games for post-fall rehabilitation; secondly, we present the haptic chair system and the principles of living lab design; thirdly, we report on a preliminary study conducted to check for feasibility, acceptance and safety of the system; fourthly, we describe the system and show how older adults' involvement drove the design; lastly, we conclude and propose ideas for future work.

\section{RELATED WORK}

Rehabilitation is more effective if the prescribed exercises are performed on a regular basis, over a long time period. In this regard, the patients' motivation to perform their rehabilitation activities for as long as necessary is a key success factor. Many authors have studied the use of game mechanics to increase patients' motivation to perform rehabilitation tasks, such as works in [20] or [21]. A literature survey was done by Rego et al., who proposed a classification of rehabilitation games [22]. Due to the large body of literature on the subject, we focus here on recent works related to falls.

Uzor et al. have worked on the participatory design of a post-fall rehabilitation game for older adults [23]. The authors propose a design methodology that involves older adults in the concept stages of the design process. The methodology consists of conducting design workshops in small groups (3 or 4 participants at a time). The workshops are organized in 4 phases: discussion of past experiences, scenarios and personas, a game session and user sketches. Thanks to this method, the key factors that limit adherence to the currently used at-home rehabilitation tools could be identified. In addition, they could help people from the target user group coming up with game design ideas for a set of rehabilitation activities. In a followup work, the same team has used their findings to implement and test a serious game for fall rehabilitation [24]. They found that the resulting design overcomes the major limitations of standard care and is usable by and acceptable for the end users. Finally, in a third paper, Uzor and Baillie studied the long-term use of their exergame in ecological conditions, with people that suffered one or several falls. They show that it yields better adherence to the rehabilitation program than the standard care tools [25]. This work is a very good example of what we would like to achieve in this project. It stresses the importance of "empowering" [23] older adults, by involving them from the ideation phase of the project, to maximize usability, acceptance and adherence to the rehabilitation program.

Another work by Proffitt and Lange focused on the usercentered design of exercise games for older adults to prevent falls [26]. To design a new exercise game, they first performed a focus group to inform the design. The implementation of the game was then done iteratively, in a user testingimplementation cycle. Following this method, they also produced a design that older adults enjoyed and found easy to use.

Other authors have studied the design constraints of games for muscle strength and balance rehabilitation to prevent successive falls. In [27], the authors have evaluated three stepping exergames for fall prevention with a group of 14 older adults. In this study, Skæjret et al. proposed an evaluation method based on 7 game elements and 5 movement characteristics to provide design insights for such exergames. Lastly, Ejupi et al. have worked on the design and evaluation of a fall risk selfassesment system including inertial sensors and a Microsoft Kinect sensor [28]. The evaluation was done in ecological conditions (the system was installed in people's homes). The study involved 62 participants in 3 countries over 4 months. The observations showed feasibility of such a system but the authors reported shortcomings, which, we believe, could have been avoided by using a co-design approach.

To the best of our knowledge, all efforts to develop serious games or systems relying on game technology tackling the issue of falls in older adults have focused on muscle strength and balance for rehabilitation after a fall or for prevention. Our approach to this problem is original as it addresses the psychological consequences of falls and not the physical issues. In addition, other works have focused on people who live independently at home whereas our system targets clinical applications.

\section{CO-DESIGN APPROACH FOR A HAPTIC CHAIR}

In this section we present the haptic chair and the living lab co-design methodology we adopted.

\section{A. The haptic chair}

The moving seat system was originally created, by the BackWell company [29]. It aims to prevent lumbago back pain in workers who sit at their desk all day, by mobilizing the upper-body muscles through the movement of the seat, activated periodically, say 5 minutes every half-hour. The chair has a mechanical system moved by a single motor to mimics the movement of the hips during walking. The motor and actuators are placed under the chair's seat. An animation can be seen on the manufacturer's website [29].

Combining this chair with our virtual strolling program has two interests: firstly, it may enhance the virtual strolling experience by adding proprioceptive sensations to the visual and auditory sensations of the game secondly; the walklike movement stimulates the motor scheme (brain activities involved in motor control). We chose this chair because it 


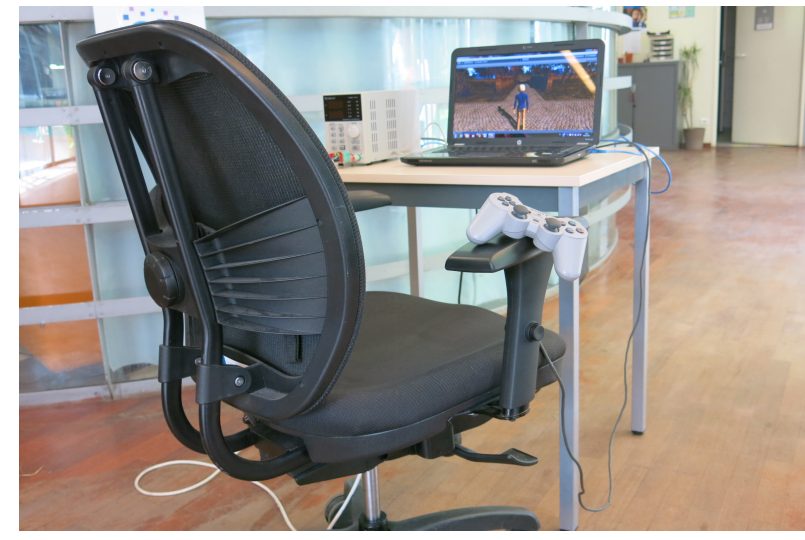

Fig. 1. Picture of the Virtual Promenade system setup.

is an affordable and easy to repurpose solution, as speed can easily be controlled by varying the the motor's input voltage. A picture of the chair and the whole Virtual Promenade system is presented on Figure 1.

\section{B. The living lab design approach}

It is now well accepted in the game-design community that iterative design practices with early, extensive playtesting are essential to make a good game [30]. Moreover, co-design approaches involving all relevant stakeholders are strongly recommended for the creation of tools for people with disabilities [31]. Finally, thorough scientific evaluation at multiple stages in the design process is essential in healthcare-oriented applications, to ensure harmlessness and efficacy. The living lab methodology [17], which combines these three components, thus appeared most suitable to design our system, a medical game for people with temporary or permanent disabilities.

The living lab approach rests on five basic principles:

- Openness: We took care to involve as many parties as possible in the design process, including patients, caregivers, experts, and anyone who expressed genuine interest.

- Influence: Each opinion was recorded and taken into account on equal footing with others, to avoid overemphasizing "expert" recommendations over end-user feedback.

- Reality: We tested our solution often and in the most realistic setting possible, with actual patients of Broca hospital in Paris, France and field healthcare practitioners with "normal" knowledge of such tools (as opposed to the rehabilitation technology experts involved in the earliest stages).

- Value creation: We were careful to systematically check that any technological upgrade, new feature or other modification did increase value for one or more stakeholders, in order to avoid technological push. Note that we mean value as defined by the stakeholders, which therefore does include, but is not limited to, commercial value.

- Sustainability: Although the project is in its early stages and sustainability is not really an issue yet, we took steps to ensure it by "repurposing" easily available, moderately priced parts (a chair designed to treat back pain, consumer grade game controllers, free software etc.). Our system is thus affordable and easy enough to repair to be deployed and maintained in many contexts, from small rural rehabilitation offices to large city hospitals.

This paper describes the first phases of this iterative, participative process, starting with harmlessness study.

\section{ACCEPTANCE AND FEASIBILITY STUDY}

We started our design by conducting a single-center, community-based cohort survey to explore the patients' acceptance and tolerance of VRE and of the moving seat. The secondary goal of this study was to evaluate the technical and organizational feasibility of the kind of intervention we propose to treat psycho-motor consequences of falls through safe, seated activities.

The study involved 8 patients, 4 males and 4 females. All patients were hospitalized in our geriatric acute or subacute care units. The participants' ages ranged from 81 to 94 (mean $=87.4 \pm 4.17)$ and their Mini Mental State Examination (MMSE) scores ranged from 20 (mild cognitive impairment) to 30 (normal cognitive functioning). The patients had a MMSE score of $25.8 \pm 3.5$, on average. Five of the participants reported a fear of falling; six had a history of repeated falls; and two were depressed. Lastly, all of them had a moderate or severe gait disorder ( 5 moderate, 3 severe). All subjects gave a written informed consent to participate in the study.

\section{A. The moving seat}

1) Protocol: To test the moving seat system, participants were simply asked to sit on the equipped chair, for 5 to 20 minutes per session. Each participant had between three and five sessions on the chair; speed varied across sessions. All tested three speeds: slow walk (37.5 cycles per minute), normal walk (50 to 70 cycles per minute) and fast walk (100 cycles per second). The reason why some patients did not have all five sessions ( 3 had three sessions and 1 had four) is because, during the study, their care schedule was not compatible with the activity sessions schedule, they were too tired or sick or were not willing to proceed to the session.

The evaluation was performed by administrating a satisfaction questionnaire to the participants after each session.

2) Results: All participants except one gave a positive (very positive for 2 of them) global feedback about the chair. All participants felt safe and said the seat's movement felt good. Only one out of eight patients did not enjoy the activity. Finally, all but one (the one who did not enjoy the activity and had a negative impression) said they were willing to continue the activity after each session.

The seat's movement did not cause pain to any of the participants; neither did it cause them nausea. These results show a strongly positive feedback of the participants and good acceptance and tolerance. Lastly, all participants favored the medium speed and one of them said the fast speed made her feel like she was running. 


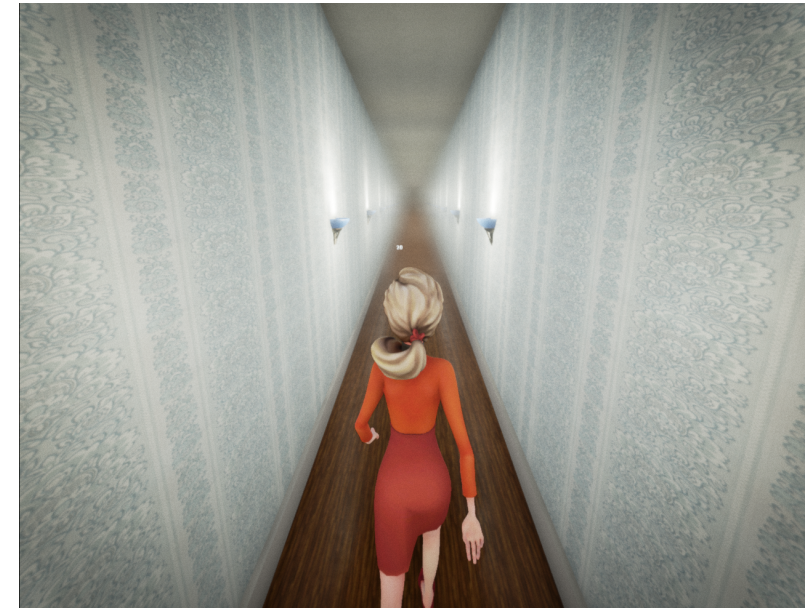

Fig. 2. Screen capture of the software used for the preliminary study

\section{B. Virtual reality training}

1) Protocol: The same patients also participated in an evaluation of the virtual reality exposure. The equipment for the sessions consisted of a standard computer, a video projector, a flight simulator-like joystick and 3D game software developed using the Unreal Engine. They were asked to use the joystick to control a virtual avatar walking in a very long corridor. They had to reach the end of the corridor. The avatar could only walk forward or backward. The subject had 5 minutes to make his or her avatar walk as far as possible; if they achieved the goal, by traveling the maximum distance, the session ended before the time was up. To increase difficulty and attempt to provoke fear of falling, the corridor widened as the avatar traveled through it, to start with a seemingly safer environment, as the walls are close, and progressively make it more plausible to fall, with the walls being further from the avatar. A screen shot of the software is presented on Figure 2

At the conclusion of each session, the participants filled a satisfaction questionnaire and the travel distance was recorded. The distance traveled, its evolution across sessions and the number of sessions to achieve the goal are as many objective evaluation criteria of the task's difficulty. The questionnaires inform about subjective acceptance.

2) Results: 7 out of 8 subjects gave positive feedbacks on the activity and only one found it rather unpleasant. The same ratio was observed regarding the visual aspect of the virtual environment; only one of the participants found it unpleasant, the same that did not appreciate the activity. However, four participants expressed frustration that they could not move their avatar sideways to freely stroll in the environment and that they would appreciate an outdoor location more. One of the participants even expressed anxiety because of the walls being too close at the beginning of the simulation. In addition, two of the subjects complained about the lack of realism. They said that the character walked too fast, that it walked backwards instead of turning around when they pulled the joystick and that it gave the impression that the character was staying in place while the environment moved in the opposite direction.

Five of the subjects reported that performing this activity gave them an impression of walking. The same participants answered that they were satisfied by this activity. However, none of the subjects reported that this activity triggered anguishing feelings, linked to their fear of falling. Lastly, all participants thought the joystick was easy to use.

Regarding the traveled distances, the average over three sessions increased from $128.7 \pm 30.8 \mathrm{~m}$ (mean \pm standard deviation) at the first session to $140.0 \pm 5.8 \mathrm{~m}$ at the third session. This represents a $11.2 \pm 5.8 \mathrm{~m}(8.7 \%)$ increase. However, we did not find any significant correlation between the performance of the subjects and their initial fear of falling, nor did we find significant differences related to cognitive status or gender. This shows that the software and controls are easy enough for use by people with mild cognitive impairment.

\section{Observations}

During this preliminary evaluation, we attempted to identify technical, procedural and organizational factors that could influence the use of our system in a hospital rehabilitation care unit. Regarding the technical factors, the main issue is related to leg fractures: people who suffered such injuries cannot stand up or lay their foot on the ground. However, hospitals are usually equipped with people lifters, used to transfer people from a bed to a wheelchair, and the chair's height can be adjusted so that the patient's feet do not touch the ground.

Regarding the protocol, the main issue we observed is patients being tired or not motivated. The fatigue issue can be addressed by having breaks during the activity. The motivational issue may be addressed by improving the content of the activity using game mechanics, as it was shown that they may improve intrinsic motivation in rehabilitation-related tasks [21].

Lastly, organizational issues are linked to fatigue of patients, care schedules and incontinence of some patients. The first two issues are easily solved by including the VRE activities in the care schedule. This is eased by the automated nature of the system; a therapist could handle more than one patient at the time if equipment and room are sufficient. Incontinence issues are not an obstacle to feasibility, as they are very common in geriatric care, and already integrated in the standard procedures.

Overall, both main elements of the system, the moving seat and the software, were evaluated positively, are well tolerated and accepted by the patients. In addition, there does not seem to be any serious obstacle to deployment in a rehabilitation care context.

We observed that people were quite sensitive to the environment's design and that it could impact people's impression on the game and motivation to continue the activity. In addition, none of these activities provoked fear of falling in the subjects. According to the specialists, fear of falling arises mostly when people go outside their home. Both issues may be addressed 


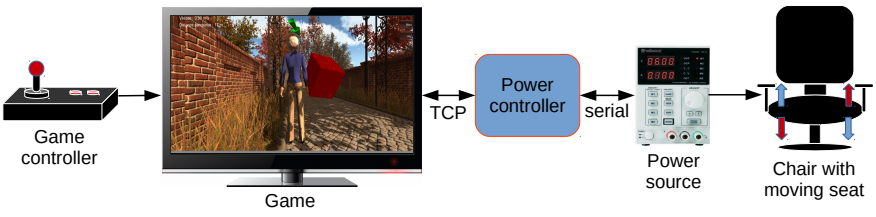

Fig. 3. The Virtual Promenade system.

at the same time by offering outdoor virtual environments. Moreover, this led us to work on synchronizing the haptic chair with the game and adding sounds to improve presence, which was shown to play an important role in provoking anxiety feelings in VRE [32]. This is described in the following section.

\section{The Virtual Promenade System}

Our approach consists in treating the traumas due to falls similarly to PTSDs, as explained before, using a serious game with proprioceptive sensory feedback. To avoid unnecessary work and reduce costs (which is essential for future deployment), we used commercially available elements, so we could focus solely on the game and movement sensory feedback design.

We adopted a user-centered living lab design approach, as described in Section III-B. The early version of Virtual Promenade only consisted in a virtual outdoor environment, a model of a modern city, in which the player controlled a virtual character to stroll freely in a third-person view mode. We chose a city environment for three reasons: cities are particularly stressful environments; the users asked for an outdoor environment in the preliminary study; and, according to the experts, the fear of falling mostly arises when older adults go outside, whereas they usually feel safe at home.

To increase immersion, we connect the game to the chair with the moving seat described earlier. The goal of our work being to propose a seated activity to reduce fear of falling in elderly patients who suffer post-fall syndromes, we deliberately chose to use a classic game controller for human-machine interaction rather than an interaction device that would require the user to perform physical tasks. We experimented with several types of game controllers, as detailed in sections V-C and VI.

The Virtual Promenade system is composed of a game controller, the virtual strolling game, the haptic chair, a programmable power source (connected to the motor, to control its input voltage) and a power controller program that receives commands from the game through TCP and sends commands to the power source through a serial-over-USB protocol. This setup is represented in Figure 3. A picture of the system is presented in Figure 1. In the three following sections, we describe the key elements in more detail.

\section{A. Controlling the armchair from the game}

As described in Section III-A, the moving seat system is lead by a voltage-controlled electrical motor. The motor's input voltage is regulated thanks to a Velleman PS3005D programmable laboratory power source. The goal is to synchronize the speed of the chair's seat movement with the player's avatar in the virtual strolling game. This is achieved through TCP client-server communication between the game and the power control program, which, in turn, sends voltage control commands to the power source through serial-overUSB communication.

\section{B. The virtual strolling game}

For our fast prototyping purposes, the game was designed using the Unity integrated game development environment. This tool combines the advantages of being quite comprehensive, user-friendly, cost-effective and of having multi-platform release capabilities. Our character models were designed using Autodesk Character Creator, a tool allowing to quickly create ready-for-use character models. The walk animations and the environment models were downloaded for free or purchased on the Unity Asset Store, a community-based content sharing platform provided by Unity. Lastly, the sound effects were downloaded on free sound effect exchange platforms.

The game is quite simple. The player controls a human avatar, whose appearance can be selected among 8 models, and has to stroll through a virtual environment. The strolling can be either free or guided through the instructions of a therapist, or through objects (large blinking cubes) that the player has to collect. When collectable objects are involved, a 3D green arrow at the top of the view helps players find the objects by pointing in the direction of the next object to collect. Once the player has picked-up a cube, the next cube appears. If the player follows the path correctly, the next cube appears in sight from the location of the one he or she has just collected. Lastly, the view can be selected among first-person view and third-person view at any time.

The game starts with an interactive tutorial and has three levels with increasing difficulty:

- The tutorial takes place in an outdoors empty space with sand-like floor and a few far trees (to give visual orientation cues). It explains how to control the character, the mechanics of the game when pick-up objects are involved and how to switch between first and third-person views. The instructions are given in text bubbles.

- The first level takes place in an autumn forest path, in which the player can stroll freely. The purpose of this level is mostly to give the player some time to get acquainted with the controls without goal constraints.

- The second level is a park. This time, the player has to stay on a paved path with turns and collect the cubes.

- The last level is a city environment. This level also has collectable cubes and introduces cars, spawning randomly, in the player's vicinity, following the roadways, as moving obstacles.

A screen capture of the game in the Park environment is presented on Figure 4 


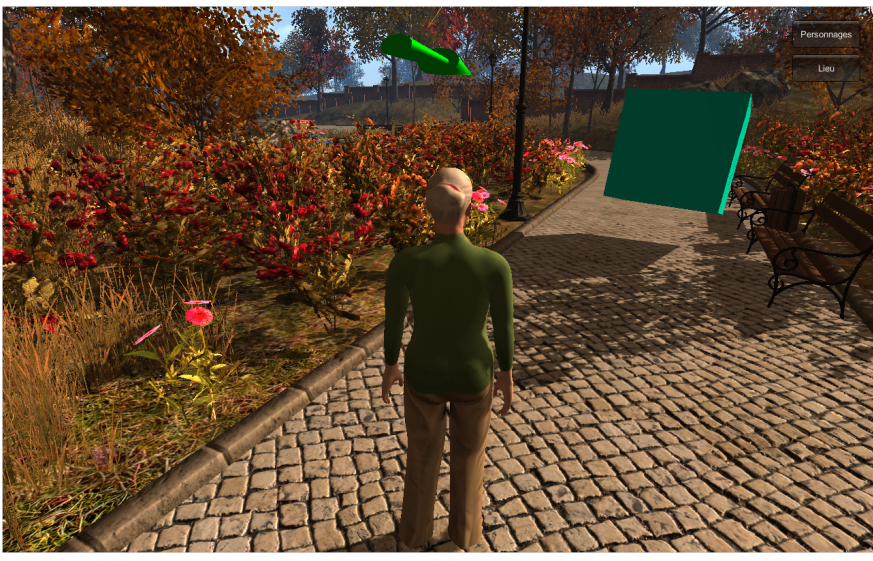

Fig. 4. Screen capture of the Virtual Promenade game in the Park environment.

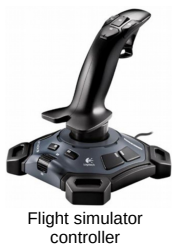

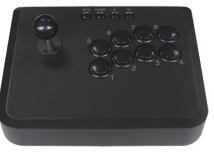

Arcade-like controller

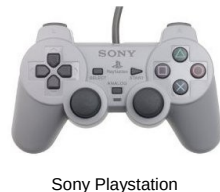

controller

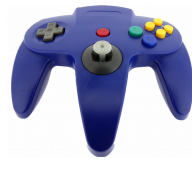

Nintendo 64 controller
Fig. 5. Pictures of the game controllers we experimented with.

\section{The game controllers}

Our aim was to find a game controller that is easy to use by older adults who are not familiar with this type of device. We thus experimented with several, commercially available game controllers:

- a Logitech flight simulator-like joystick;

- a Mayflash arcade game controller;

- a Sony Playstation controller;

- a Nintendo 64 controller.

The only controls in use during the game are the analog stick and one button to change the view from third-person to first-person and back. We first experimented with the flight simulator joystick but it had flaws, as described in Section VI-B. We then experimented with the other controllers shown on Figure 5. Characters for the avatar and levels can be selected by a researcher or a therapist, so they can keep control on the sessions' progress.

\section{DISCUSSION}

During the design phase, we mostly tested with healthy older adults, as a proxy to our target users, and one patient who was hospitalized after a fall. The participants were recruited on a voluntary basis. Some are "expert users" who frequently participate in activities of our living lab. All participants were women over 80 years old. In total, 8 people participated in the trials, but one of them did not want to try the system herself and only gave her opinion based on observing two other participants play.

We had three formal trial sessions but also a few informal trials with one or two participants at the time, after they had participated in another activity at the living lab. In the last trial session, participants had to fill a satisfaction questionnaire with 15 questions in blocks of 5 . The first block was about the tutorial and the ease of use of the controls; the second and third blocks were about the environments and the feeling of immersion.

The remainder of this section sums-up our observations and highlights the design choices we made in the design phase.

\section{A. Virtual environments, avatars and view}

We started off with only one environment, a model of a Japanese city, as a city environment was recommended by the experts and we had noted in the feasibility study that people asked for an outdoor environment. Only one of the 4 participants that tried the city environment liked it. The others said that it felt cold and unwelcoming. The participant who liked this environment said it was only because, as an urban resident, she could relate to this environment. We thus added two environments: an autumn forest and a park full of trees and flowers. All of the 5 participants who tried the game with these environments were very satisfied with them and said they were realistic enough.

Regarding the avatars, we only had one character model at first, a thin lady with gray short hair. Participants in the first trial complained that they did not identify with this character because its body shape looked nothing like a real elderly person. We thus added 7 new character models. Our models include 5 female characters and 3 male. We made them so that their body shape was more realistic, according to what our participants recommended. All subjects seemed satisfied with the choice of character models. One person even said she identified well with the character she chose.

Lastly, we experimented with two views: first-person and third-person. In the first trial, we only had the third-person view. The subjects complained that the camera was too close to the character, which made it harder for them to see where they were going. We changed the camera's position to be higher and further from the character. We did not have other complaints in the other trials. We also added a first-person view. We observed that people performed better in controlling their avatar in first-person view, but 4 out of 5 subjects said that they preferred the third-person view because it was more reassuring. We think that keeping both options is interesting because the third-person view is reassuring and the first-person view yields better immersion, and may elicit increased anxiety, which could be useful in the later stages of VRE therapy.

\section{B. Game controllers}

The flight-simulator joystick did not turn out to be adapted in the first trial. The subjects said it was too hard to maneuver, and it required a table to put it on, which was not practical. Indeed, the addition of two control axes and the use of a heavier joystick (for more stability) made the use of this type of controller more difficult. In addition, in the preliminary phase, the game and the haptic chair were mostly tested 
separately, which prevented from identifying the problem regarding joystick placement.

The Sony Playstation game controller was too difficult to use as it required fine motor control of the thumbs and older adults are not used at all to that kind of device. Walking in a strait line turned out to be particularly difficult for our participants.

The arcade controller was quite promising and had two main advantages: it can be put on the user's lap and it is big enough so the user can rest his or her arm on it. In addition it has big buttons that users successfully used to navigate the instructions in the tutorial. However, it has a major flaw: the directional stick is made with all-or-nothing switches, which doesn't allow the player to control the speed of the avatar.

Lastly, the Nintendo 64 controller was used successfully. It is lightweight and practical, as the player can hold the handle in the middle with one hand and use the analog stick with the other hand (which the participants did), by grabbing the stick between the thumb and the index finger. The analog stick also has a guide that helps going straight. However, this device is obsolete and can have the same drawbacks as the Sony Playstation controller, though it is a bit easier to use, as it still requires fine motor control.

This issue is very important. One of the subjects even reported spontaneously that controlling the character being too difficult for her hindered her experience as she could not concentrate on the walking sensation. Facing this issue, a good practice is to support several controllers for adaptability to as many patients as possible.

\section{Haptic chair and immersion}

The feedback on the immersion was very positive. One participant out of eight said she did not think the haptic chair enhanced the experience. All other participants said they were feeling a good walking sensation. However, two subjects thought that it would be better if their feet were moved as well and one said that the walking sensation was reduced at low speed. This means that the synchronization between the chair's mechanism and the movement of the on-screen character may require some fine tunning.

\section{Tutorial}

The tutorial was only introduced in the third session of trials. All 4 participants went through it successfully. However, they required help because they kept moving the character while trying to read the following instructions and could not do both at the same time. This issue could be solved by freezing the avatar when the instructions text is displayed so they cannot move their avatar while reading.

\section{CONCLUSION AND FUTURE WORK}

We introduced a novel method to treat PFS in older adults through virtual reality exposure. We started by evaluating the feasibility and patient tolerance to this kind of intervention. We showed that the system was tolerated well, as it did not cause any motion sickness or pain and found no obstacles to its applicability in a hospital care environment. We then built a prototype of a virtual strolling system, following a user-centered co-creation methodology. Thanks to that design approach, we came close to a truly adapted design, though some improvement are still required.

In our test sessions, we observed that older adults are sensitive to the visual layout of the game and we identified it as an important motivational factor. We observed that the combination of visual, auditory and proprioceptive sensations yields a good level of immersion, especially when using firstperson view. However, we could not find the ideal controller for older adults.

To improve our design, we would like to try using a motorized wheelchair joystick, because we could tie it to the armrest of the chair and its ergonomics has already been thoroughly studied. We would also like to add a city environment that people would appreciate, with non-playable characters and cars, to raise the level of difficulty and yield anxiety in people who have PFS.

In our future work, we will attempt to improve the game mechanics and explore the possibilities offered by the addition of a virtual therapist in the game. In addition, we would like to add a pressure sensor on the backrest of the chair, which would be used to prevent the character from moving if patients put their back against the chair, thus forcing them to hold their back while they play. This could allow to put more emphasis on the muscular reinforcement aspects of the system. Finally, we will conduct a clinical evaluation of this system.

\section{ACKNOWLEDGMENT}

The authors would like to thank Région Île-de-France and EPHAD Ressources for funding this project and Dr. Pierre Jouvelot for his helpful advice.

\section{REFERENCES}

[1] R. Marks, "Physical activity and hip fracture disability: a review," Journal of aging research, vol. 2011, 2011.

[2] J. Murphy and B. Isaacs, "The post-fall syndrome," Gerontology, vol. 28, no. 4, pp. 265-270, 1982.

[3] M. Gaudet, B. Tavernier, F. Mourey, C. Tavernier, D. Richard, and J. Marot, "Le syndrome de régression psycho-motrice du vieillard," Médecine et hygiène, vol. 44, no. 1656, pp. 1332-1336, 1986.

[4] F. Mourey, P. Manckoundia, I. Martin-Arveux, B. Tavernier-Vidal, and P. Pfitzenmeyer, "Psychomotor disadaptation syndrome," Geriatrics, vol. 59, no. 5, 2004.

[5] R. P. Bhala, J. O'Donnell, and E. Thoppil, "Ptophobia: Phobic fear of falling and its clinical management," Physical therapy, vol. 62, no. 2, pp. 187-190, 1982.

[6] M. E. Tinetti, C. F. M. De Leon, J. T. Doucette, and D. I. Baker, "Fear of falling and fall-related efficacy in relationship to functioning among community-living elders," Journal of gerontology, vol. 49, no. 3, pp. M140-M147, 1994.

[7] D. S. Moore and R. Ellis, "Measurement of fall-related psychological constructs among independent-living older adults: a review of the research literature," Aging and mental health, vol. 12, no. 6, pp. 684699, 2008.

[8] American Psychiatric Association, Diagnostic and Statistical Manual of Mental Disorders (DSM-5®). American Psychiatric Pub, 2013.

[9] M. C. Chung, K. J. McKee, C. Austin, H. Barkby, H. Brown, S. Cash, J. Ellingford, L. Hanger, and T. Pais, "Posttraumatic stress disorder in older people after a fall," International journal of geriatric psychiatry, vol. 24, no. 9, pp. 955-964, 2009. 
[10] F. Bloch, M. Blandin, R. Ranerison, Y. Claessens, A. Rigaud, and G. Kemoun, "Anxiety after a fall in elderly subjects and subsequent risk of developing post traumatic stress disorder at two months. a pilot study," The journal of nutrition, health \& aging, vol. 18, no. 3, pp. 303-306, 2014

[11] N. Jayasinghe, M. A. Sparks, K. Kato, K. Wyka, K. Wilbur, G. Chiaramonte, P. S. Barie, M. S. Lachs, M. O’Dell, A. Evans et al., "Posttraumatic stress symptoms in older adults hospitalized for fall injury," General hospital psychiatry, vol. 36, no. 6, pp. 669-673, 2014.

[12] F. Bloch, A.-S. Rigaud, and G. Kemoun, "Virtual reality exposure therapy in posttraumatic stress disorder: A brief review to open new opportunities for post-fall syndrome in elderly subjects," European Geriatric Medicine, vol. 4, no. 6, pp. 427-430, 2013.

[13] K. Laver, S. George, S. Thomas, J. Deutsch, and M. Crotty, "Cochrane review: virtual reality for stroke rehabilitation." European journal of physical and rehabilitation medicine, vol. 48, no. 3, pp. 523-530, 2012.

[14] M. K. Holden, "Virtual environments for motor rehabilitation: review," Cyberpsychology \& behavior, vol. 8, no. 3, pp. 187-211, 2005.

[15] Y. Baram and A. Miller, "Virtual reality cues for improvement of gait in patients with multiple sclerosis," Neurology, vol. 66, no. 2, pp. 178-181, 2006.

[16] M. Gerardi, J. Cukor, J. Difede, A. Rizzo, and B. O. Rothbaum, "Virtual reality exposure therapy for post-traumatic stress disorder and other anxiety disorders," Current psychiatry reports, vol. 12, no. 4, pp. 298 305, 2010.

[17] B. Bergvall-Kareborn and A. Stahlbrost, "Living lab: an open and citizen-centric approach for innovation," International Journal of Innovation and Regional Development, vol. 1, no. 4, pp. 356-370, Jan. 2009.

[18] M. Agogué, G. Comtet, P. Le Masson, J.-F. Menudet, and R. Picard, "Managing innovative design within the health ecosystem: the living lab as an architect of the unknown," Revue Management et Avenir Santé, vol. 1, no. 1, pp. 17-32, Oct 2013.

[19] M. Pino, S. Benveniste, R. Picard, and A.-S. Rigaud, "User-driven innovation for dementia care in France: The LUSAGE living lab case study," Interdisciplinary Studies Journal, vol. 3, no. 4, pp. 251-268, 2014.

[20] E. Flores, G. Tobon, E. Cavallaro, F. I. Cavallaro, J. C. Perry, and T. Keller, "Improving patient motivation in game development for motor deficit rehabilitation," in Proceedings of the 2008 International Conference on Advances in Computer Entertainment Technology. ACM, 2008, pp. 381-384.

[21] M. Mihelj, D. Novak, M. Milavec, J. Ziherl, A. Olenšek, and M. Munih, "Virtual rehabilitation environment using principles of intrinsic motivation and game design," Presence, vol. 21, no. 1, pp. 1-15, Feb 2012.
[22] P. Rego, P. M. Moreira, and L. P. Reis, "Serious games for rehabilitation: A survey and a classification towards a taxonomy," in Information Systems and Technologies (CISTI), 2010 5th Iberian Conference on. IEEE, 2010, pp. 1-6.

[23] S. Uzor, L. Baillie, and D. Skelton, "Senior designers: empowering seniors to design enjoyable falls rehabilitation tools," in Proceedings of the SIGCHI Conference on Human Factors in Computing Systems. ACM, 2012, pp. 1179-1188.

[24] S. Uzor and L. Baillie, "Exploring and designing tools to enhance falls rehabilitation in the home," in Proceedings of the SIGCHI Conference on Human Factors in Computing Systems, ser. CHI '13. New York, NY, USA: ACM, 2013, pp. 1233-1242.

[25] _ , "Investigating the long-term use of exergames in the home with elderly fallers," in Proceedings of the 32nd Annual ACM Conference on Human Factors in Computing Systems, ser. CHI '14. New York, NY, USA: ACM, 2014, pp. 2813-2822.

[26] R. Proffitt and B. Lange, "User Centered Design and Development of a Game for Exercise in Older Adults," The International Journal of Technology, Knowledge, and Society, vol. 8, no. 6, pp. 95-112, Mar. 2013.

[27] N. Skjæret, A. Nawaz, K. Ystmark, Y. Dahl, J. L. Helbostad, D. Svanæs, and B. Vereijken, "Designing for movement quality in exergames: Lessons learned from observing senior citizens playing stepping games," Gerontology, vol. 61, no. 2, pp. 186-194, 2015.

[28] A. Ejupi, Y. J. Gschwind, T. Valenzuela, S. R. Lord, and K. Delbaere, "A kinect and inertial sensor-based system for the self-assessment of fall risk: A home-based study in older people," Human-Computer Interaction, no. just-accepted, 2015.

[29] (2015, Sep.) Backwell website. [Online]. Available: http://back-well.com

[30] K. Salen and E. Zimmerman, Rules of play: Game design fundamentals. MIT press, 2004.

[31] (2015, Oct.) Active and assisted living programme documents and resources. [Online]. Available: http://www.aal-europe.eu/documentsresources/

[32] M. Price, N. Mehta, E. B. Tone, and P. L. Anderson, "Does engagement with exposure yield better outcomes? components of presence as a predictor of treatment response for virtual reality exposure therapy for social phobia," Journal of anxiety disorders, vol. 25, no. 6, pp. 763-770, 2011. 